\title{
Predicting Elective Orthopaedic Sports Medicine Surgical Cancellations Based on Patient Demographics
}

\author{
Brandon Petrone, D.O., Jordan Fakhoury, D.O., Prashant Matai, M.B.B.S., \\ Adam Bitterman, D.O., Randy M. Cohn, M.D., and Larry Lutsky, Ph.D.
}

\begin{abstract}
Purpose: To evaluate whether patient demographics are associated with cancellation of elective orthopaedic sports medicine surgical procedures. Methods: We retrospectively reviewed the electronic medical records of 761 patients who were scheduled to undergo an elective sports medicine orthopaedic operation from January 1, 2015, to December 31, 2017. The patients were divided into 2 groups: those who underwent the scheduled procedure (group A) and those in whom the operation was canceled for any reason prior to the surgical date and not rescheduled (group B). Univariate analysis assessed patient factors consisting of age, sex, race, language, marital status, occupation status, type of insurance (Medicaid or Medicare vs private), smoking history, employment status, and history of surgery to determine which demographic factors led to an increased risk of elective case cancellation. Results: Patients who canceled were significantly older (46.5 years vs 41.5 years, $t=2.432, P=.015)$ than those who do not. In addition, current smokers $(22.5 \%$ vs $10.9 \%$, $\left.\chi^{2}=10.85, P=.001\right)$, patients with Medicare or Medicaid versus private insurance $\left(16.7 \%\right.$ vs $10.0 \%, \chi^{2}=5.35, P=$ $.021)$, non-English-speaking patients $\left(29.5 \%\right.$ vs $\left.11.6 \%, \chi^{2}=11.43, P=.001\right)$, and patients without a history of surgery requiring anesthesia $\left(18.8 \%\right.$ vs $\left.9.6 \%, \chi^{2}=9.96, P=.002\right)$ were all more likely to cancel. When all studied variables were examined in a logistic regression analysis, of the above demographic variables, only insurance status was no longer significant, given its correlation with age and language. Conclusions: Increased age ( $\geq 46.5$ years), non-English speaking, smoking, lack of a history of surgery requiring anesthesia, and Medicaid or Medicare insurance were found to contribute to an increased risk of elective orthopaedic surgery cancellation. Level of Evidence: Level III, case-control study.
\end{abstract}

$\mathbf{O}$ perative care is one of the major areas of health care service provided by hospitals, with ambulatory orthopaedic surgery being one of the most common procedures performed ${ }^{1}$ and one of the largest sources of revenue for a hospital facility. ${ }^{2}$ However, it has also made orthopaedics one of the specialties undergoing the most cancellations in the hospital today.

From the Zucker School of Medicine at Hofstra/Northwell, Plainview Hospital (B.P., J.F., P.M., A.B., R.M.C.), Plainview; and Krasnoff Quality Management Institute (L.L.), New Hyde Park, New York, U.S.A.

The authors report that they have no conflicts of interest in the authorship and publication of this article. Full ICMJE author disclosure forms are available for this article online, as supplementary material.

Received June 7, 2019; accepted November 18, 2019.

Address correspondence to Brandon Petrone, D.O., Zucker School of Medicine at Hofstra/Northwell, Plainview Hospital, 888 Old Country Rd, Plainview, NY 11803,U.S.A.E-mail: bop03862@gmail.com

(C) 2019 THE AUTHORS. Published by Elsevier Inc. on behalf of the Arthroscopy Association of North America. This is an open access article under the CC BY-NC-ND license (http://creativecommons.org/licenses/by-nc-nd/4.0/). 2666-061X/19703

https://doi.org/10.1016/j.asmr.2019.11.004
Elective orthopaedic surgical cancellation rates typically range from $10 \%$ to $34 \%,{ }^{3-5}$ which vary depending on the country and patient population. These cancellations can be related to multiple factors associated with the patient, surgeon, or hospital, but most cancellations have been shown to be patient related and avoidable. . $6,7^{-1}$

Cancellation of elective surgical procedures is attributed to several factors. These include nonattendance, socioeconomic factors, improper medical workup, lack of preoperative fasting, and hospital-related causes. ${ }^{8,9}$ Of these, patient-related factors remain one of the most common reasons for case cancellation., ${ }^{3,8,9}$ Cancellation of elective surgical cases negatively impacts patient experience; surgeon scheduling; and overall operating room function, efficiency, and revenue. $^{10-13}$ Even with advancements in hospital quality measures directed toward decreasing patient cancellation rates, these "avoidable" cancellations continue to occur. ${ }^{8,14,15}$

Despite the increased interest in surgical cancellations, knowledge regarding the factors that influence 
Table 1. Univariate Analysis Comparing Patient Demographic Characteristics and Cancellation Rate

\begin{tabular}{|c|c|c|c|c|c|c|}
\hline Demographic Factor & $\mathrm{n}$ & Mean (SD) & $\%$ Canceled & $\chi^{2}$ & $t$ Test & $P$ Value* \\
\hline Sex & & & & 0.091 & & .763 \\
\hline Female & 240 & & 12.9 & & & \\
\hline Male & 319 & & 13.8 & & & \\
\hline Married & 226 & & 12.4 & & & \\
\hline Single & 330 & & 13.3 & & & \\
\hline Occupation & & & & 0.072 & & .788 \\
\hline Smoking status & & & & 10.855 & & $.001^{*}$ \\
\hline Nonsmoker & 431 & & 10.9 & & & \\
\hline Smoker & 120 & & 22.5 & & & \\
\hline Insurance & & & & 5.351 & & $.021^{*}$ \\
\hline Private insurance & 299 & & 10.0 & & & \\
\hline Medicaid or Medicare & 258 & & 16.7 & & & \\
\hline Language & & & & 11.430 & & $.001^{*}$ \\
\hline English speaking & 507 & & 11.6 & & & \\
\hline Non-English speaking & 44 & & 29.5 & & & \\
\hline Surgical history & & & & 9.961 & & $.002^{*}$ \\
\hline History of surgery & 324 & & 9.6 & & & \\
\hline No history of surgery & 234 & & 18.8 & & & \\
\hline Age & & & & & 2.432 & $.015^{*}$ \\
\hline Did not cancel & 484 & $41.49 \mathrm{yr}(16.66 \mathrm{yr})$ & & & & \\
\hline Canceled & 75 & $46.51 \mathrm{yr}(16.49 \mathrm{yr})$ & & & & \\
\hline
\end{tabular}

SD, standard deviation.

*Statistically significant $(P<.05)$.

patient cancellations is incomplete. Currently, there are limited data on determining which patients are at high risk of elective orthopaedic surgery cancellation. A more complete understanding of patient factors that influence these cancellations can enable better insight into identifying high-risk patients and may allow physicians to implement preoperative interventions to decrease potentially avoidable cancellations. The purpose of this study was to evaluate whether patient demographics are associated with cancellation of elective orthopaedic sports medicine surgical procedures. Our hypothesis was that patient demographics would play a significant role in cancellation of elective surgery.

\section{Methods}

Because of the retrospective nature of this study, institutional review board review was waived at our institution. We retrospectively reviewed the electronic

Table 2. Parameter Estimates for Logistic Regression Model

\begin{tabular}{|c|c|c|c|c|c|c|}
\hline Variable & B & SE & Wald & $d f$ & Significance & $\operatorname{Exp}(\mathrm{B})$ \\
\hline$\overline{\text { Age }}$ & 0.031 & 0.010 & 8.817 & 1 & $.003^{*}$ & 1.031 \\
\hline Sex & 0.100 & 0.296 & 0.115 & 1 & .734 & 1.106 \\
\hline Marital status & 0.222 & 0.328 & 0.458 & 1 & .498 & 1.249 \\
\hline Smoking status & 0.835 & 0.305 & 7.481 & 1 & $.006^{*}$ & 2.305 \\
\hline Insurance provider & 0.516 & 0.320 & 2.597 & 1 & .107 & 1.675 \\
\hline Race code & & & 0.163 & 2 & .922 & \\
\hline Language & 1.007 & 0.474 & 4.520 & 1 & $.034^{*}$ & 2.738 \\
\hline Surgical history & 1.007 & 0.314 & 13.506 & 1 & $<.001^{*}$ & 3.172 \\
\hline Constant & -4.560 & 0.690 & 43.655 & 1 & $<.001$ & 0.010 \\
\hline
\end{tabular}

Race 1, comparison of white vs black; Race 2, comparison of white vs other races (Hispanic, Asian, multiracial); SE, standard error.

*Statistically significant $(P<.05)$. 
Fig 1. History of surgery versus cancellation rate. (CI, confidence interval.)

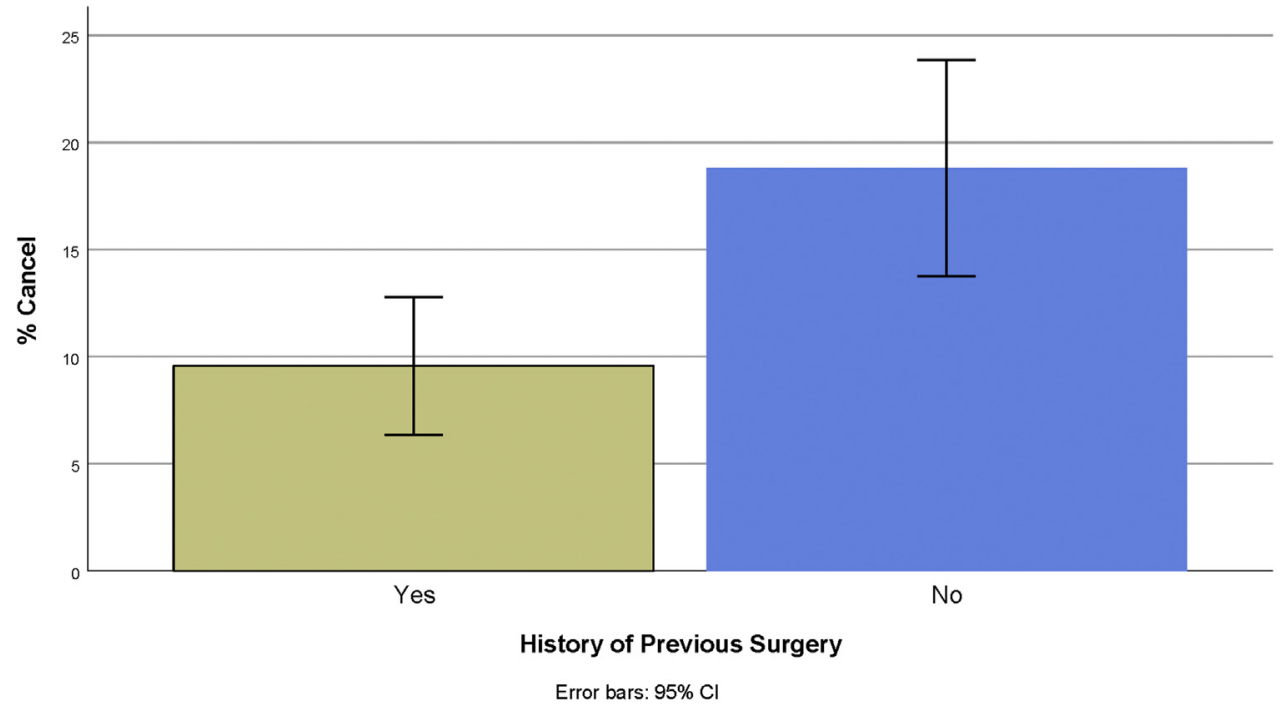

medical records of 761 patients who were scheduled to undergo an elective sports medicine orthopaedic operation performed by a board-certified orthopaedic sports medicine surgeon (R.M.C.) at a single academic community hospital from January 1, 2015 to December 31, 2017. All patients seen on an outpatient basis and scheduled to undergo an elective orthopaedic surgical procedure were included in this study. Patients undergoing revision surgery, those undergoing inpatient procedures such as total joint replacement, and those requiring operations considered urgent or emergent such as fracture repair or irrigation and debridement were excluded from the completed-case group. Cancellations owing to surgeon- or hospital-related factors were also excluded. Patient demographic variables including age, sex, race, language, marital status, occupation status, type of insurance (Medicaid or Medicare vs private), smoking history, employment status, history of surgery requiring anesthesia, and procedure type were recorded. A patient with a surgical history was defined as any patient undergoing a surgical procedure requiring sedation with or without intubation in an ambulatory or operating room setting.

\section{Statistical Analysis}

Univariate analysis assessed which demographic factors led to an increased risk of elective case cancellation (Table 1). Significant predictors in the univariate models including age, smoking history, primary language, and history of surgery were used to create a predictive power model for patient cancellation (Table 2). By use of this model, the predictive probability of cancellation was calculated for different values of age, primary language, smoking history, and history of surgery (Table 2). Significance was assessed at $P<.05$. To evaluate whether the scheduled procedure played a role in the cancellation rate, we performed a cross tabulation between the scheduled procedure and cancellation rate; procedures with fewer than 10 patients were excluded. We then used a $\chi^{2}$ analysis to determine whether there was a significant difference in the cancellation rate between the 2 groups.

\section{Results}

During the study period, the patients were divided into 2 groups: those who underwent the scheduled procedure (672 patients) (group A) and those in whom the operation was canceled for any reason prior to the surgical date and not rescheduled (89 patients) (group B). A total of 571 patients (486 in group $A$ and 85 in group $B$ ) met the inclusion criteria, and their demographic factors were recorded (Table 1). Overall, $13.4 \%$ of patients canceled prior to or on the day of the scheduled surgical procedure and did not reschedule. Surgical history was found to be significantly associated with surgical cancellation: Patients with no surgical history requiring anesthesia were more likely to cancel $\left(18.8 \%\right.$ vs $9.6 \%, \chi^{2}=9.96$, $P=.002$ ) (Fig 1). Current smokers were more likely to cancel than were nonsmokers or previous smokers $\left(22.5 \%\right.$ vs $\left.10.9 \%, \chi^{2}=10.85, P=.001\right)$ (Fig 2). Patient primary language was also found to be significantly associated with surgical cancellation, with non-Englishspeaking patients more likely to cancel ( $29.5 \%$ vs $11.6 \%$, $\chi^{2}=11.43, P=.001$ ) (Fig 3). Patients who canceled were significantly older (46.5 years vs 41.5 years, $t=2.432$, $P=.015$ ) than those who did not (Fig 4). Finally, patients with Medicare or Medicaid were more likely to cancel than were patients with private insurance $(16.7 \%$ vs $10.0 \%, \chi^{2}=5.35, P=.021$ ) (Fig 5). When all studied variables were examined in a logistic regression analysis, only insurance status was no longer significant, given its 


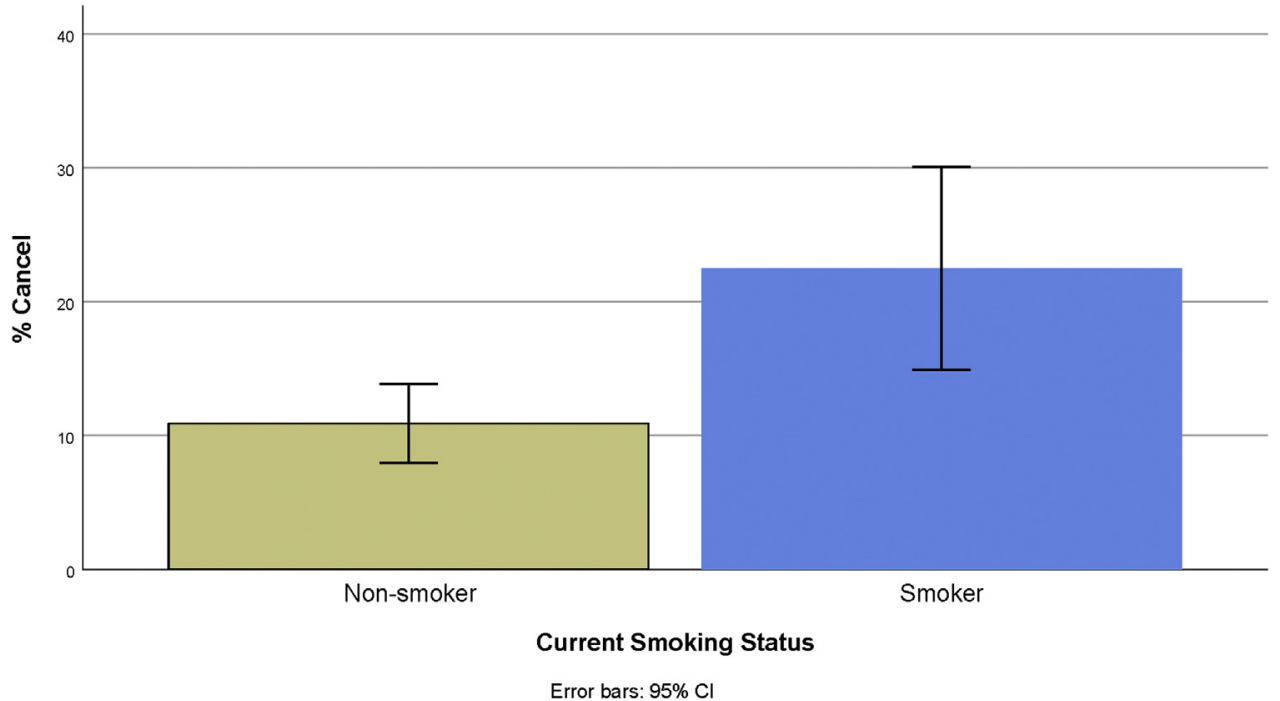

Fig 2. Smoking status versus cancellation rate. (CI, confidence interval.) correlation with age and language (Table 2). When we analyzed the scheduled procedures between the 2 groups, there were 5 procedures that were scheduled to be performed in more than 10 patients (Table 3). The $\chi^{2}$ analysis revealed no significant difference between the type of scheduled procedure and the rate of cancellation (Table 4).

The predictive model identified age $(\mathrm{B}=0.031$, $P=.003)$, smoking status $(B=0.835, P=.006)$, nonEnglish speaking $(\mathrm{B}=1.007, P=.034)$, and lack of a surgical history requiring anesthesia $(\mathrm{B}=1.154, P=$ .0002 ) as the top predictors of cancellation (Table 2). By use of this model, a 42-year-old patient with no risk factors has only a $3.9 \%$ chance of cancellation. In contrast, a 54-year-old male smoker who speaks Spanish and has no surgical history has a $52.7 \%$ chance of canceling (Table 5).

\section{Discussion}

The results of this study show that patient demographic factors including age, smoking status, non-English speaking, and no surgical history requiring anesthesia led to an increased rate of elective orthopaedic sports surgery cancellation. No difference was found between scheduled procedure and the rate of cancellation.

Most elective surgery cancellations have been found to be patient related. ${ }^{3-9}$ Socioeconomic concerns related to symptomatic improvement during the waiting period, fear of surgery, economic burden, and
Fig 3. Primary language versus cancellation rate. (CI, confidence interval.)

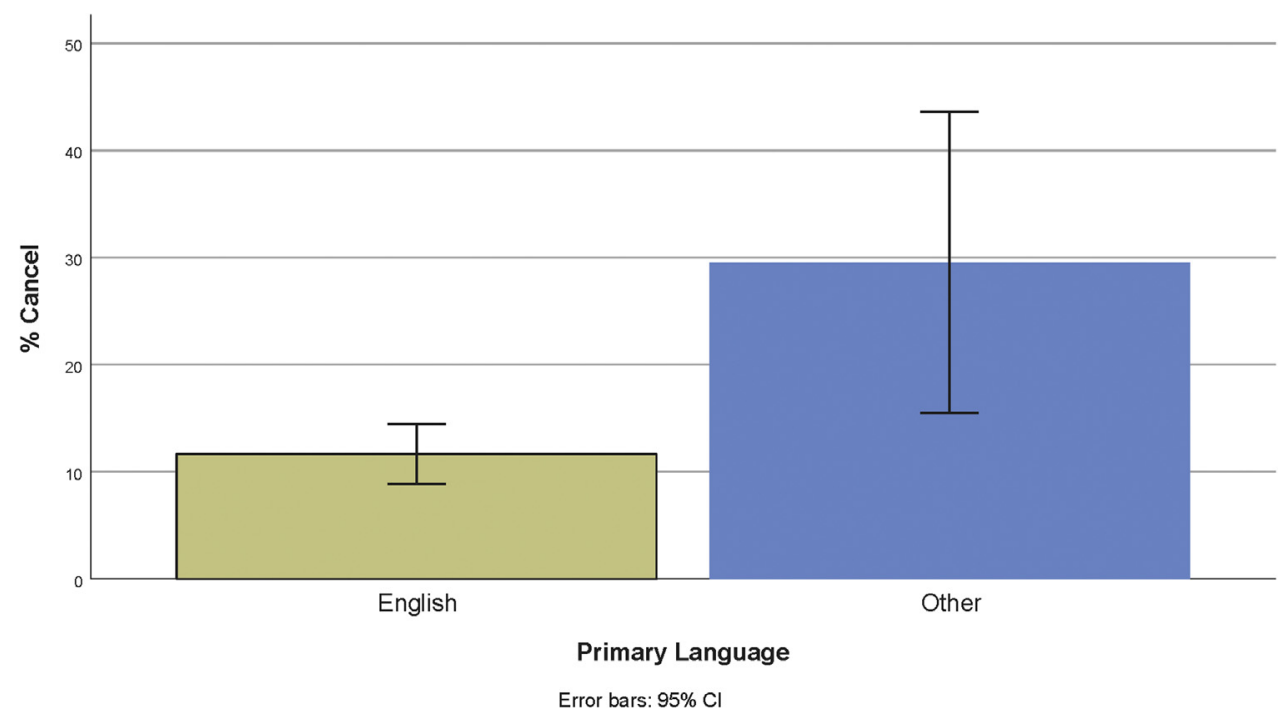


Fig 4. Cancellation rate versus mean age (in years). (CI, confidence interval.)

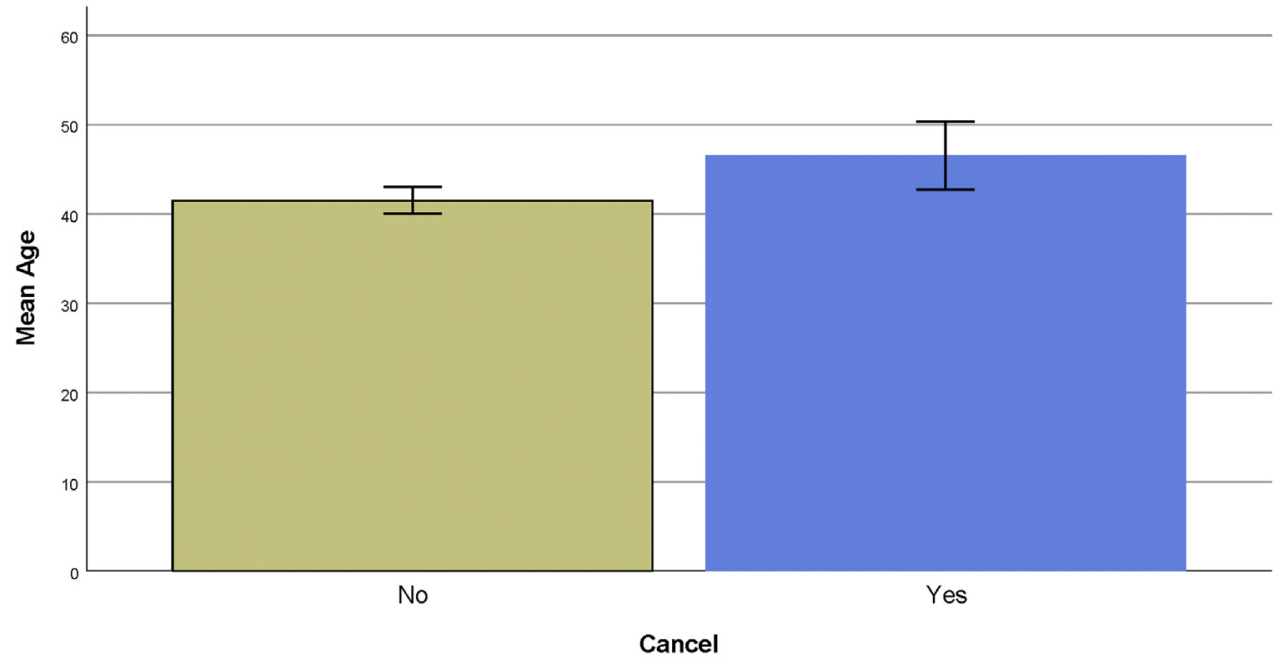

Error bars: $95 \% \mathrm{Cl}$ postoperative recovery are among the most common reasons. ${ }^{6,7}$ Caesar et al. ${ }^{8}$ performed a root-cause analysis of elective orthopaedic surgical cancellations and concluded that patient cancellations were a result of patient self-cancellation (17\%), social reasons associated with family or work (16\%), and inadequate preoperative assessment such as medical clearance or preoperative fasting $(12 \%)$. Every cancellation in our study was patient related and not related to the surgeon or the hospital.

Yoo et al. ${ }^{6}$ performed a study analyzing patients with a diagnosed rotator cuff tear who decided not to undergo surgery. The main reason for patient cancellation was improvement of symptoms. Gong et al. ${ }^{7}$ surveyed patients as to why they did or did not undergo elective carpal tunnel surgery. They found that the main reason for undergoing surgery was the fear of symptomatic progression. Patients who canceled stated that symptomatic improvement had occurred. Our findings do not support these results because we found no difference in cancellations based on the scheduled procedure, indicating that there are other patient-related factors contributing to elective orthopaedic sports surgery cancellation. Gong et al. and Yoo et al. also showed that fear of the surgical procedure was a factor contributing to elective surgery cancellation. We were able to show that patients who had no history of surgery were more likely to cancel. We believe that patients who have undergone a prior surgical procedure would have less fear or anxiety when scheduling another operation.

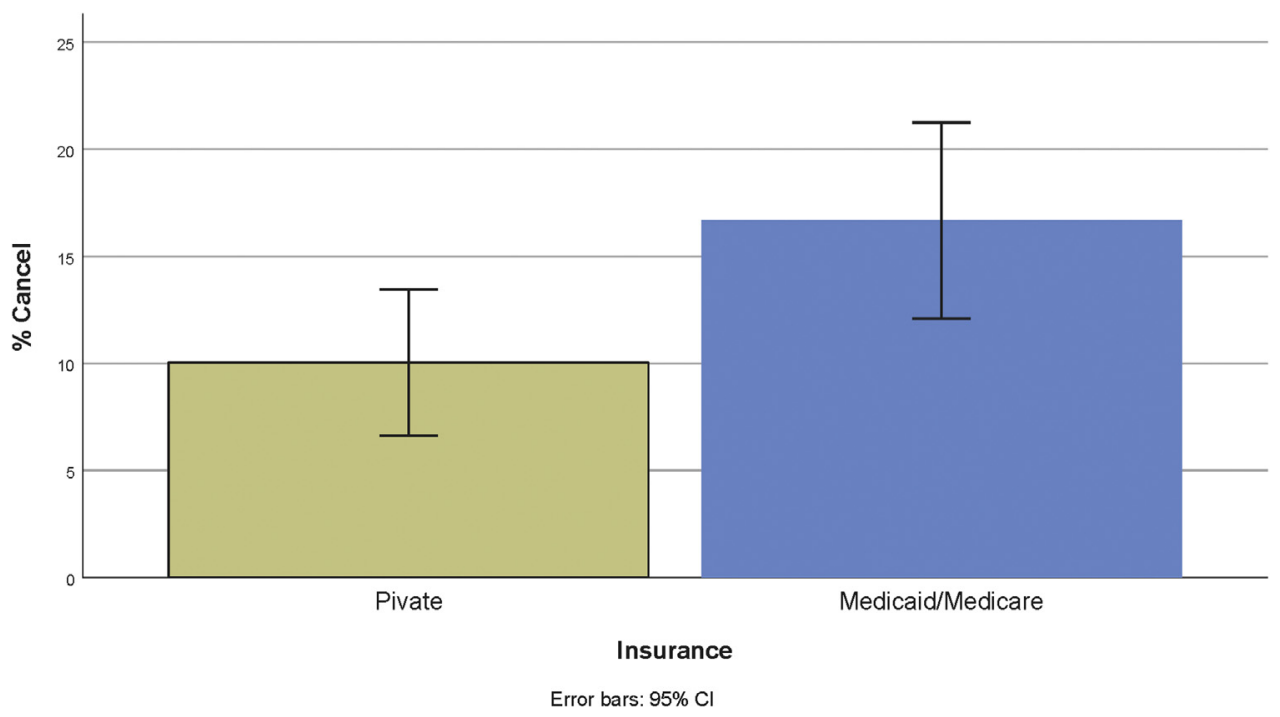

Fig 5. Insurance provider versus cancellation rate. (CI, confidence interval.) 
Table 3. Cross Tabulation of Cancellation Rate by Surgical Procedure

\begin{tabular}{|c|c|c|c|}
\hline & \multicolumn{2}{|c|}{ Canceled } & \multirow[b]{2}{*}{ Total } \\
\hline & No & Yes & \\
\hline \multicolumn{4}{|l|}{ Scheduled procedure } \\
\hline \multicolumn{4}{|l|}{ Distal biceps repair } \\
\hline Patients, n & 15 & 2 & 17 \\
\hline$\%$ within scheduled procedure category & 88.2 & 11.8 & 100.0 \\
\hline \multicolumn{4}{|l|}{ Hip arthroscopy } \\
\hline Patients, n & 75 & 11 & 86 \\
\hline$\%$ within scheduled procedure category & 87.2 & 12.8 & 100.0 \\
\hline \multicolumn{4}{|l|}{ Knee arthroscopy } \\
\hline Patients, n & 124 & 16 & 140 \\
\hline$\%$ within scheduled procedure category & 88.6 & 11.4 & 100.0 \\
\hline \multicolumn{4}{|l|}{ Knee arthroscopy and ACL repair } \\
\hline Patients, n & 78 & 11 & 89 \\
\hline \% within scheduled procedure category & 87.6 & 12.4 & 100.0 \\
\hline \multicolumn{4}{|l|}{ Shoulder arthroscopy } \\
\hline Patients, n & 152 & 31 & 183 \\
\hline$\%$ within scheduled procedure category & 83.1 & 16.9 & 100.0 \\
\hline \multicolumn{4}{|l|}{ Total } \\
\hline Patients, n & 444 & 71 & 515 \\
\hline$\%$ & 86.2 & 13.8 & 100.0 \\
\hline
\end{tabular}

Clear communication is essential for orthopaedic surgeons to explain the preoperative instructions, procedural details, postoperative recovery process, and surgical alternatives while discussing treatment options with patients. Language barriers for non-Englishspeaking patients can disrupt this communication between the patient and the surgeon. With the use of professional interpreters, significant improvements have been made in patients' explanations of their symptoms, patient satisfaction, and the ability to obtain informed consent. ${ }^{16,17}$ However, these improvements did not eliminate the language disparity, ${ }^{16}$ and our data support this issue.

Cancellation of elective orthopaedic surgical procedures is a significant problem that can lead to decreased operating room efficiency and revenue for a hospital. ${ }^{10}$ Turunen et al. ${ }^{11}$ recently analyzed the average cost to a hospital in Finland for elective dayof-surgery cancellations across multiple surgical subspecialties. Orthopaedic surgery cancellation was associated with one of the highest costs to the hospital compared with other surgical subspecialties. The average cost was $\$ 2,713(€ 2,415)$ per patient. This number is estimated to be half the cost of cancellation in the United States. ${ }^{11}$

Several improvements in quality of care and systemsbased practice focusing on limiting elective surgical cancellations have been found to be successful. Sebach et al. ${ }^{18}$ described an evidence-based nurse practitioner-led preoperative evaluation clinic within a large orthopaedic practice. They analyzed elective surgery cancellation rates and missed charges due to cancellations before and after implementation of the nurse practitioner-led preoperative evaluation clinic. The rate of surgical cancellations was significantly decreased from $7.7 \%$ to $0.8 \%(P=.007)$ and lost revenue decreased from $\$ 386,033$ to $\$ 184,480$ after implementation. Singhal et al. ${ }^{15}$ performed a prospective study analyzing patient-related factors leading to same-day elective orthopaedic surgery cancellations. With the use of a telephone questionnaire administered 1 week before the scheduled elective procedure specifically aimed at addressing patient-related factors leading to cancellation, the rate decreased from $10 \%$ to $1.6 \%(P=.01)$. At our institution, on the day before the scheduled surgical procedure, the patient is contacted by a registered nurse to explain the preoperative protocol and what time to arrive.

Using patient demographics, we can now attempt to risk stratify patients to determine their overall probability of cancellation. This analysis will allow physicians and their staff to focus on foreseeable cancellations and implement new patient-specific strategies to improve patient satisfaction, quality of care, and systems-based practice and to save lost revenue. In addition, understanding which patients are more likely to cancel will allow improved allocation of time and resources, with a greater focus on high-risk patients.

\section{Limitations}

There are some limitations to this study. This study was retrospective in nature and all data reviewed were taken from electronic medical records, which can limit discoverable information. In addition, this study was limited to a single orthopaedic sports surgeon at a single institution (a community hospital). Therefore, the results may not be generalizable to other orthopaedic subspecialties and patient populations. Finally, we did not differentiate between patients who canceled ahead of time and those who canceled on the day of the scheduled procedure or assess the reasons why each patient canceled.

\section{Conclusions}

Increased age ( $\geq 46.5$ years), non-English speaking, smoking, lack of a history of surgery requiring anesthesia, and Medicaid or Medicare insurance were found to contribute to an increased risk of elective orthopaedic surgery cancellation.

Table 4. $\chi^{2}$ Analysis Between Scheduled Surgical Procedure and Cancellation Rate

\begin{tabular}{lccc}
\hline & Value & $d f$ & Asymptotic Significance (2-Sided) \\
\hline Pearson $\chi^{2}$ & $2.469^{*}$ & 4 & .650 \\
Likelihood ratio & 2.424 & 4 & .658 \\
No. of valid cases & 515 & & \\
\hline
\end{tabular}

${ }^{*}$ One cell $(10.0 \%)$ had an expected count of less than 5 . The minimum expected count was 2.34 . 
Table 5. Example Case Scenarios Using Significant Patient Demographic Characteristics

\begin{tabular}{lclllr}
\hline & Age, yr & Smoking Status & Language & History of Surgery & Predicted Cancellation Risk, \% \\
\hline Case example 1 & 42 & No & English & Yes & 3.7 \\
Case example 2 & 54 & Yes & Spanish & No & 52.7 \\
\hline
\end{tabular}

NOTE. A 42-year-old English-speaking patient who is a nonsmoker and has a surgical history has only a 3.7\% risk of cancellation. In contrast, a 54-year-old Spanish-speaking patient who smokes and has no surgical history has a $52.7 \%$ risk of cancellation.

\section{References}

1. Steiner CA, Karaca Z, Moore BJ, Imshaug MC, Pickens G. Surgeries in hospital-based ambulatory surgery and hospital inpatient settings, 2014: Statistical brief \#223. In: Healthcare Cost and Utilization Project (HCUP) statistical briefs. Rockville, MD: Agency for Healthcare Research and Quality, 2017;1-12.

2. Merritt Hawkins. Physician inpatient/outpatient revenue survey. Available at: https://www.merritthawkins.com/ uploadedFiles/MerrittHawkins_RevenueSurvey_2019.pdf 2019. Accessed May 24, 2019.

3. Desta M, Manaye A, Tefera A, et al. Incidence and causes of cancellations of elective operation on the intended day of surgery at a tertiary referral academic medical center in Ethiopia. Patient Saf Surg 2018;12:25.

4. Schofield WN, Rubin GL, Piza M, et al. Cancellation of operations on the day of intended surgery at a major Australian referral hospital. Med J Aust 2005;182: 612-615.

5. Dhafar KO, Ulmalki MA, Felemban MA, et al. Cancellation of operations in Saudi Arabian hospitals: Frequency, reasons and suggestions for improvements. Pak J Med Sci 2015;31:1027-1032.

6. Yoo JC, Lim TK, Kim DH, Koh K-H. Comparison between the patients with surgery and without surgery after recommendation of surgical repair for symptomatic rotator cuff tear. J Orthop Sci 2018;23:64-69.

7. Gong HS, Baek GH, Oh JH, Jeon SH, Chung MS. Factors affecting willingness to undergo carpal tunnel release. J Bone Joint Surg Am 2010;91:2130-2136.

8. Caesar U, Karlsson J, Olsson L-E, Samuelsson K, HanssonOlofsson E. Incidence and root causes of cancellations for elective orthopaedic procedures: A single center experience of 17,625 consecutive cases. Patient Saf Surg 2014;8:24.
9. Mesmar M, Shatnawi NJ, Faori I, Khader YA. Reasons for cancellation of elective operations at a major teaching referral hospital in Jordan. East Mediterr Health J 2011;17: 651-655.

10. Childers CP, Maggard-Gibbons M. Understanding costs of care in the operating room. JAMA Surg 2018;153:e176233.

11. Turunen E, Miettinen M, Setälä L, VehviläinenJulkunen K. Financial cost of elective day of surgery cancellations. J Hosp Adm 2018;7:30.

12. Leslie RJ, Beiko D, van Vlymen J, Siemens DR. Day of surgery cancellation rates in urology: Identification of modifiable factors. Can Urol Assoc J 2013;7:167-173.

13. Ivarsson B, Kimblad PO, Sjberg T, Larsson S. Patient reactions to cancelled or postponed heart operations. J Nurs Manag 2002;10:75-81.

14. Olson RP, Dhakal IB. Day of surgery cancellation rate after preoperative telephone nurse screening or comprehensive optimization visit. Perioper Med (Lond) 2015;4:12.

15. Singhal R, Warburton T, Charalambous CP. Reducing same day cancellations due to patient related factors in elective orthopaedic surgery: Experience of a centre in the UK. J Perioper Pract 2014;24:70-74.

16. Lindholm M, Hargraves JL, Ferguson WJ, Reed G. Professional language interpretation and inpatient length of stay and readmission rates. J Gen Intern Med 2012;27:1294- 1299.

17. Lee JS, Pérez-Stable EJ, Gregorich SE, et al. Increased access to professional interpreters in the hospital improves informed consent for patients with limited English proficiency. J Gen Intern Med 2017;32:863-870.

18. Sebach AM, Rockelli LA, Reddish W, Jarosinski JM, Dolan CL. Development of a nurse practitioner-managed preoperative evaluation clinic within a multispecialty orthopedic practice. J Nurse Pract 2015;1 1:869-877. 